\title{
OTIMIZAÇÃO DA DETERMINAÇÃO DE ÁCIDO FÓLICO EM LEITES ENRIQUECIDOS ATRAVÉS DA ANÁLISE DE SUPERFÍCIE DE RESPOSTA ${ }^{1}$
}

\author{
Rodrigo Ramos CATHARINO2,*, Helena Teixeira GODOY²
}

\begin{abstract}
RESUMO
A etapa de extração é uma das maiores fontes de erros na determinação de vitaminas, já que estas são compostos freqüentemente lábeis e estão presentes em concentrações muito pequenas numa matriz orgânica complexa que são os alimentos. A extração desenvolvida por Catharino e Godoy para análise de ácido fólico em leites enriquecidos consiste em uma técnica rápida e bastante simples, sendo por esse motivo escolhida neste estudo para ser avaliada. A análise de superfície de resposta foi aplicada para investigar as alterações na concentração de ácido fólico em leite enriquecido frente a algumas modificações, especialmente, na etapa de extração. Foram avaliados quatro parâmetros: quantidade de amostra, tempo de extração, volume de ácido tricloroacético (TCA) e tempo de espera para injeção no equipamento. As condições preestabelecidas para análise de $1,0 \mathrm{~g}$ amostra, 10 minutos no ultra-som, $350 \mu \mathrm{L}$ de ácido tricloroacético e injeção imediata são parâmetros que estão dentro da faixa teórica ótima, segundo os resultados obtidos pela análise da superfície de resposta, embora quantidades um pouco inferiores de amostra $(0,9 \mathrm{~g})$ e maior volume de TCA $(425 \mu \mathrm{L})$ proporcionaram quantidades de ácido fólico ligeiramente superiores.
\end{abstract}

Palavras-chave: ácido fólico; leites enriquecidos; análise multivariada.

\section{SUMMARY}

OPTIMIZING OF FOLIC ACID DETERMINATION IN ENRICHED MILK BY RESPONSE SURFACE ANALYSIS. The extracting stage is one of the biggest origin of mistakes in determining vitamins, once those are frequently sensitive compounds and are found in small concentrations in a complex organic matrix, or in other words, foods. The extraction step developed by Catharino \& Godoy to folic analysis in enriched milk consists of a very simple and quick technique, which is why it was chosen in this study to be evaluated. Response surface analysis was applied to investigation modification in concentration of folic acid in enriched milks with some changes, specially, in extraction step. Four parameters have been evaluated: sample quantity, time for extraction, volume of tricloroacetic acid (TCA) and waiting time for injection in the equipment. The pre-establish conditions for analysis of $1,0 \mathrm{~g}$ sample, 10 minutes in ultrasound, $350 \mu \mathrm{L}$ of tricloroacetic acid and immediate injection are parameters in a theoretical optimum average, according to the results obtained by response surface analysis, although inferior quantities of sample and larger volume of TCA $(425 \mu \mathrm{L})$ give a slightly superior quantity of folic acid.

Keywords: folic acid; enriched milks; surface analysis.

\section{1 - INTRODUÇÃO}

O ácido fólico(AF) é uma vitamina do complexo $B$, muito importante para a manutenção do organismo

\footnotetext{
${ }^{1}$ Recebido para publicação em 29/01/00. Aceito para publicação em 13/08/01.

${ }_{2}^{2}$ Faculdade de Engenharia de Alimentos. C.P. 621. CEP 13083970.E-mail: robit@bol.com.br

${ }^{*}$ A quem a correspondência deve ser enviada.
}

humano. Acredita-se que a deficiência de AF possa acarretar desde malformações congênitas no feto (espina bífida, encefalocelece, fenda palatina e hidrocefalia) até doenças cardiácas [2, 4, 5, 6, 10, 13, 14, 15, 16, 18].

Atualmente a maioria dos países que sofrem com doenças acometidas pela falta de ácido fólico estão suplementando os alimentos com esta importante vitamina. Vários produtos enriquecidos com AF começam a ser lançados no mercado, visando auxiliar na prevenção e no controle das doenças causadas pela deficiência dessa vitamina.

Nos últimos anos várias metodologias analíticas foram desenvolvidas para a determinação e quantificação de folatos em alimentos, principalmente utilizando a cromatografia líquida de alta eficiência $[3,7,9,11,12$, $17,19,20]$.

Apenas DONG, LEPORE, TARUMOTO [8] realizaram um extensivo trabalho observando as respostas de tempos de retenção frente a diferentes parâmetros independentes analisados, como $\mathrm{pH}$ de fase móvel, modificador orgânico e tipos de coluna para a análise de vitaminas do complexo $B$, incluindo o ácido fólico. Porém, nenhum trabalho analisou, mesmo em análise univariada, a concentração do ácido fólico frente a outros parâmetros. Não existe na literatura trabalhos que possibilitem a constatação de interações de efeitos frente a parâmetros de determinação de ácido fólico, utilizando uma metodologia multivariada de superfície de resposta.

O objetivo deste estudo foi encontrar melhores condições de extração do AF em amostras de leite enriquecido a partir do método desenvolvido por CATHARINO, GODOY [3], utilizando uma metodologia de superfície de resposta para a otimização do processo.

\section{2 - MATERIAIS E MÉTODOS}

\section{1 - Amostras}

A amostra analisada foi constituída apenas de uma marca de leite em pó enriquecida com ácido fólico $(250 \mu \mathrm{g} /$ $100 \mathrm{~g}$ ), destinado ao consumo. O produto estava dentro do prazo de validade regular e sem danos aparentes.

\section{2 - Reagentes}

O padrão de ácido fólico foi cedido pela M. CASSAB Comércio e Indústria Ltda., Santo Amaro, SP, Brasil. (SIGMA cód. F-7876, lote 40H321). A acetonitrila, grau cromatográfico, o ácido acético, o ácido tricloroacético, o ácido fosfórico e o hidróxido de potássio, grau analítico foram adquiridos da MERCK, Brasil. A água utilizada na preparo das amostras e das fases móveis foi purificada no sistema Milli-Q (MILLIPORE). As fa- 
ses móveis foram filtradas em filtros MILLIPORE, com poros de $0,45 \mu \mathrm{m}$ de diâmetro.

\section{3 - Equipamentos}

Para análise por cromatografia líquida de alta eficiência foi utilizado um cromatógrafo a líquido HP (HELWETT PACKARD) série 1100, com degaseificador, bomba quaternária, injetor automático de 1 a $100 \mu \mathrm{L}$ de capacidade. Um detector de arranjo de diodos (DAD) da HP série 1100 , todos acoplados ao software HPChemstation.

\section{4 - Métodos}

A metodologia desenvolvida por CATHARINO, GODOY [3], consiste em tomar cerca de 1,0g de amostra, previamente homogeneizada e adicionar $3 \mu \mathrm{L}$ de $\mathrm{KOH}$ $0,1 \mathrm{~mol} / \mathrm{L}$. Após 10 minutos em ultra-som adicionaramse $3 \mathrm{~mL}$ de ácido fosfórico $(0,1 \mathrm{~mol} / \mathrm{L}), 350 \mu \mathrm{L}$ de ácido tricloroacético e completou-se o volume com tampão fosfato $(\mathrm{pH} 6,5)$ para $10 \mathrm{~mL}$ em balão volumétrico. Seguiu-se a filtração em membranas Durapore (HVLP 01300 MILLIPORE), 0,45 $\mu \mathrm{m}$ de poro, antes da injeção no cromatógrafo. A vitamina foi separada em coluna Microsorb ODS-2,5 $\mu \mathrm{m}, 150 \mathrm{~mm} \times 4,6 \mathrm{~mm}$ d.i (VARIAN) protegida por uma coluna de guarda Bondesil $C_{18}, 5 \mu \mathrm{m}, 10 \times 4,6 \mathrm{~mm}$ d.i. (VARIAN).

\section{5 - Planejamento experimental fatorial e análise de superfície de resposta}

Neste estudo foram observados alguns pontos considerados críticos, como, quantidade de amostra, tempo de extração, volume de ácido tricloroacético (TCA) e tempo de espera para injeção no equipamento, na determinação do ácido fólico em leites enriquecidos, utilizando-se um delineamento estatístico fatorial completo $2^{4} \mathrm{com} 4$ variáveis independentes (Tabela 1). Para estimativa do erro experimental foram feitas 6 replicatas do experimento correspondendo aos pontos centrais. Para medir a possibilidade da não-linearidade nos valores de concentração de ácido fólico em função dos quatro fatores traçados neste experimento, oito (8) pontos axiais (alfa) foram adicionados ao planejamento no centro do experimento completo [1]. O planejamento experimental com os valores reais e codificados são apresentados na Tabela 2. A resposta $(Y)$ ou variável dependente foi a concentração de ácido fólico na amostra de leite enriquecido. As primeiras 16 linhas da Tabela 2 são suficientes para a determinação do modelo linear e são referentes ao experimento completo $2^{4}$ da linha 17 até a linha 24 do planejamento são os pontos axiais e as 6 replicatas do experimento, que são os pontos centrais, estão da linha 25 até a linha 30 .

TABELA 1. Condições preestabelecidas das variáveis independentes como nível superior (+), nível inferior (-), intermediário (0).

\begin{tabular}{lccccc}
\hline \multicolumn{1}{c}{ Varíveis } & -2 & -1 & 0 & +1 & +2 \\
\hline Peso da amostra (g) & 0,8 & 0,9 & 1 & 1,1 & 1,2 \\
Tempo de injeção (minutos) & 0 & 15 & 30 & 45 & 60 \\
Tempo no ultra-som (minutos) & 5 & 10 & 15 & 20 & 25 \\
Volume de TCA $^{a}(\mu \mathrm{L})$ & 275 & 350 & 425 & 500 & 575 \\
\hline
\end{tabular}

a TCA=ácido tricloroacético

\section{3 - RESULTADOS E DISCUSSÃO}

Verificou-se neste estudo que os efeitos estipulados na Tabela 3 são estatisticamente significativos como mostram os valores de ( $p$ ) e t de student, para a extração, embora os fatores de maior significância para o modelo foram o efeito linear do peso da amostra e o efeito quadrático de cada fator. À medida que esses valores foram aumentados a concentração de ácido fólico diminuiu, ou seja, estes fatores contribuíram negativamente para a resposta $(\mathrm{Y})$ do modelo.

TABELA 2. Resultados da concentração de ácido fólico ( $\mu \mathrm{g} /$ $100 \mathrm{~g})$ para as diversas condições de extração.

\begin{tabular}{|c|c|c|c|c|c|c|c|c|c|}
\hline \multirow[b]{3}{*}{ Ensaios } & \multicolumn{8}{|c|}{ Valores experimentais } & \multirow{3}{*}{$\begin{array}{c}\mathrm{Y}^{\mathrm{c}} \\
\mu \mathrm{g} / 100 \mathrm{~g}\end{array}$} \\
\hline & \multicolumn{4}{|c|}{ Real $^{\mathrm{a}}$} & \multicolumn{4}{|c|}{ Codificado $^{b}$} & \\
\hline & $\begin{array}{c}\text { Peso } \\
\text { (g) }\end{array}$ & $\begin{array}{c}\mathrm{ti} \\
(\mathrm{min})\end{array}$ & $\begin{array}{c}\text { tu } \\
\text { (min) }\end{array}$ & $\begin{array}{c}\text { Tca } \\
(\mu \mathrm{L})\end{array}$ & $\mathrm{X} 1$ & $\mathrm{X} 2$ & $\mathrm{X} 3$ & $\mathrm{X} 4$ & \\
\hline 1 & 0,9 & 15 & 10 & 350 & - & - & - & - & 249,51 \\
\hline 2 & 1,1 & 15 & 10 & 350 & + & - & - & - & 225,81 \\
\hline 3 & 0,9 & 45 & 10 & 350 & - & + & - & - & 232,90 \\
\hline 4 & 1,1 & 45 & 10 & 350 & + & + & - & - & 210,42 \\
\hline 5 & 0,9 & 15 & 20 & 350 & - & - & + & - & 240,70 \\
\hline 6 & 1,1 & 15 & 20 & 350 & + & - & + & - & 193,81 \\
\hline 7 & 0,9 & 45 & 20 & 350 & - & + & + & - & 210,60 \\
\hline 8 & 1,1 & 45 & 20 & 350 & + & + & + & - & 175,57 \\
\hline 9 & 0,9 & 15 & 10 & 500 & - & - & - & + & 213,99 \\
\hline 10 & 1,1 & 15 & 10 & 500 & + & - & - & + & 228,41 \\
\hline 11 & 0,9 & 45 & 10 & 500 & - & + & - & + & 231,50 \\
\hline 12 & 1,1 & 45 & 10 & 500 & + & + & - & + & 212,44 \\
\hline 13 & 0,9 & 15 & 20 & 500 & - & - & + & + & 231,62 \\
\hline 14 & 1,1 & 15 & 20 & 500 & + & - & + & + & 226,94 \\
\hline 15 & 0,9 & 45 & 20 & 500 & - & + & + & + & 248,58 \\
\hline 16 & 1,1 & 45 & 20 & 500 & + & + & + & + & 209,77 \\
\hline 17 & 0,8 & 30 & 15 & 425 & -2 & 0 & 0 & 0 & 232,75 \\
\hline 18 & 1,2 & 30 & 15 & 425 & +2 & 0 & 0 & 0 & 206,86 \\
\hline 19 & 1 & 0 & 15 & 425 & 0 & -2 & 0 & 0 & 224,13 \\
\hline 20 & 1 & 60 & 15 & 425 & 0 & +2 & 0 & 0 & 225,76 \\
\hline 21 & 1 & 30 & 5 & 425 & 0 & 0 & -2 & 0 & 221,56 \\
\hline 22 & 1 & 30 & 25 & 425 & 0 & 0 & +2 & 0 & 222,93 \\
\hline 23 & 1 & 30 & 15 & 275 & 0 & 0 & 0 & -2 & 199,54 \\
\hline 24 & 1 & 30 & 15 & 575 & 0 & 0 & 0 & +2 & 237,60 \\
\hline 25 & 1 & 30 & 15 & 425 & 0 & 0 & 0 & 0 & 253,64 \\
\hline 26 & 1 & 30 & 15 & 425 & 0 & 0 & 0 & 0 & 252,52 \\
\hline 27 & 1 & 30 & 15 & 425 & 0 & 0 & 0 & 0 & 252,76 \\
\hline 28 & 1 & 30 & 15 & 425 & 0 & 0 & 0 & 0 & 252,60 \\
\hline 29 & 1 & 30 & 15 & 425 & 0 & 0 & 0 & 0 & 254,33 \\
\hline 30 & 1 & 30 & 15 & 425 & 0 & 0 & 0 & 0 & 252,48 \\
\hline
\end{tabular}

a Peso - peso da amostra; ti - tempo de injeção; tu - tempo de extracão no ultrasom TCA - volume de ácido tricloroacético b ${ }^{b}$ De acordo com a Tabela 1. cY con centração de ácido fólico no leite.

TABELA 3. Estimativa dos efeitos, o erro puro e significância dos efeitos, $(p)$ e t de student.

\begin{tabular}{lrrrr}
\hline \multicolumn{5}{c}{ *Estimativa dos efeitos } \\
\hline \multicolumn{1}{c}{ Fator } & $\begin{array}{c}\text { Estimativa dos } \\
\text { Efeitos }\end{array}$ & \multicolumn{1}{c}{ Erro puro } & $\mathrm{t}(5)$ & $\mathrm{p}$ \\
\hline Média & 253,06 & 0,31 & 816,91 & $5,217 \mathrm{E}-14$ \\
Peso (L) & $-18,92$ & 0,31 & $-61,07$ & $2,228 \mathrm{E}-08$ \\
Peso (Q) & $-16,64$ & 0,29 & $-57,42$ & $3,03 \mathrm{E}-08$ \\
ti (L) & $-6,23$ & 0,31 & $-20,11$ & $5,622 \mathrm{E}-06$ \\
ti (Q) & $-14,07$ & 0,29 & $-48,55$ & $7,001 \mathrm{E}-08$ \\
tu (L) & $-5,47$ & 0,31 & $-17,66$ & $1,068 \mathrm{E}-05$ \\
tu 3 (Q) & $-15,42$ & 0,29 & $-53,21$ & $4,432 \mathrm{E}-08$ \\
Tca (L) & 11,59 & 0,31 & 37,41 & $2,572 \mathrm{E}-07$ \\
Tca (Q) & $-17,26$ & 0,29 & $-59,55$ & $2,526 \mathrm{E}-08$ \\
Peso com ti & $-6,94$ & 0,38 & $-18,30$ & $8,969 \mathrm{E}-06$ \\
Peso com tu & $-9,20$ & 0,38 & $-24,25$ & $2,224 \mathrm{E}-06$ \\
Peso com Tca & 10,12 & 0,38 & 26,68 & $1,384 \mathrm{E}-06$ \\
ti com tu & $-2,14$ & 0,38 & $-5,63$ & 0,0024484 \\
ti com Tca & 10,33 & 0,38 & 27,24 & $1,248 \mathrm{E}-06$ \\
tu com Tca & 15,94 & 0,38 & 42,02 & $1,44 \mathrm{E}-07$ \\
\hline
\end{tabular}

* confiança de 95\%.t de student; $p$ - significância

Os fatores que apresentaram um efeito positivo sobre a resposta $(\mathrm{Y})$ do modelo foram os que estão ligados ao volume de ácido tricloroacético ou ele apenas, provavelmente, porque o ácido auxilia na limpeza da amostra. Por último, observou-se que os efeitos negativos que contribuem em menor escala de forma absoluta, para a diminuição da variável de resposta no modelo, foram 
provenientes das variáveis tempo de injeção e tempo no banho ultra-sônico.

A Equação 1 representa o modelo experimental da extração do ácido fólico em função das variáveis estudadas.

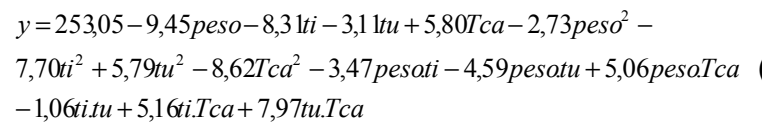

A Tabela 4 de análise de variância (ANOVA) mostra a validade do modelo pelo teste $\mathrm{F}$ e o resíduo que mostra a magnitude do erro experimental. O F tabelado $\left(F_{14,15}=2,5\right)$ foi menor que o $F$ calculado $(11,56)$, mostrando a validade do modelo experimental. $O$ valor do resíduo 985,66 foi baixo quando comparado com a regressão, e o valor do erro puro $(2,88)$ indica boa reprodutibilidade da análise.

TABELA 4. Análise de variância para desenvolvimento experimental (ANOVA).

\begin{tabular}{llllll}
\hline $\begin{array}{l}\text { Fonte de } \\
\text { variação }\end{array}$ & $\begin{array}{l}\text { Soma dos } \\
\text { quadrados }\end{array}$ & $\begin{array}{l}\text { Graus de } \\
\text { liberdade }\end{array}$ & $\begin{array}{l}\text { Quadrado } \\
\text { médio }\end{array}$ & F. calculado & F tabelado \\
\hline Regressão & 10636,94 & 14 & 759,78 & 11,56 & 2,46 \\
$\begin{array}{l}\text { Residuo } \\
\text { Falta de }\end{array}$ & 985,66 & 15 & 65,71 & & \\
ajuste & 98,78 & 10 & 98,28 & & \\
Erro puro & 2,88 & 5 & 0,576 & & \\
Total & 11622,60 & 29 & & & \\
\hline
\end{tabular}

Limite de confiança de $95 \%$.

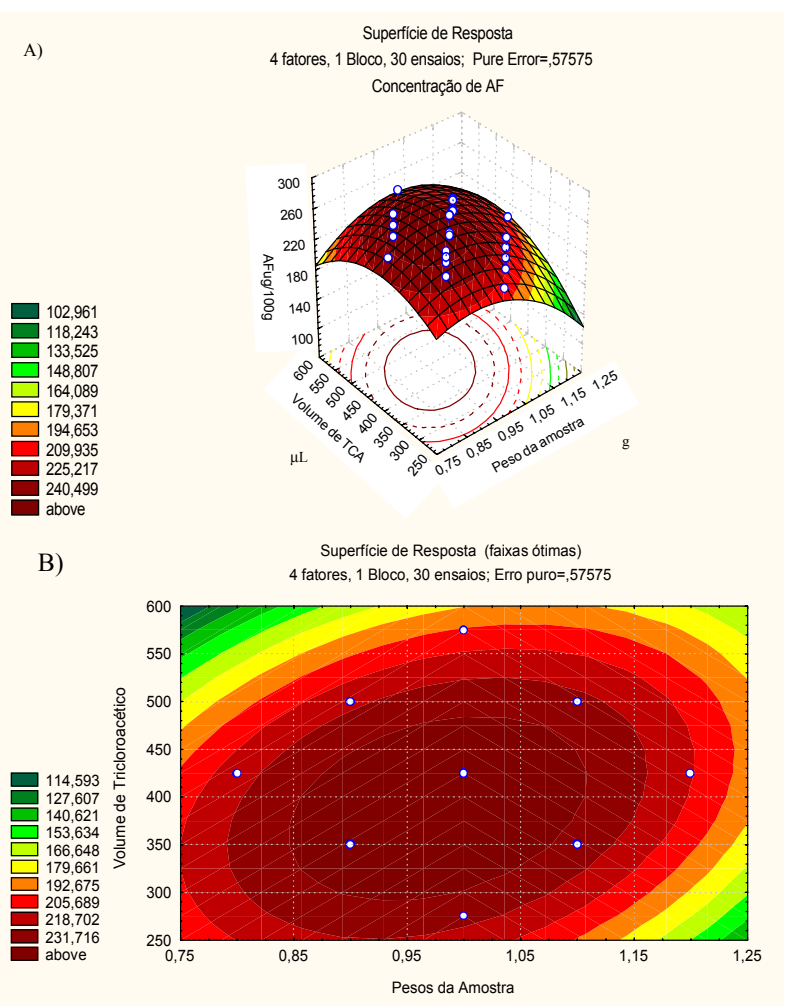

FIGURA 1. Superfície de resposta (A) e curva de contorno (B) na determinação do ácido fólico em função do volume de TCA e peso da amostra.

Neste estudo as variáveis peso da amostra e o volume de ácido tricloroacético no processo de determi- nação de AF foram as que mais se destacaram, sendo as mesmas usadas no gráfico de superfície e na curva de contorno para observação das melhores faixas de respostas (Figura 1).

A Figura 1 mostra que os ensaios no ponto central (0) foram os ótimos de resposta, ou seja, 1,0g de amostra de leite em pó, 15 minutos no ultra-som depois da adição de $3 \mathrm{~mL}$ de $\mathrm{KOH}(0,1 \mathrm{~mol} / \mathrm{L})$, adição de $425 \mu \mathrm{L}$ de ácido tricloroacético com injeção, após 30 minutos.

\section{4 - CONCLUSÕES}

Constatou-se através da análise de superfície de resposta que as faixas ótimas de trabalho para determinação da concentração de ácido fólico em leites enriquecidos foram, 0,9-1,0g de amostra de leite em pó, 10-15 minutos no ultra-som depois da adição de $3 \mathrm{~mL}$ de $\mathrm{KOH}(0,1 \mathrm{~mol} / \mathrm{L})$, adição de $350-425 \mu \mathrm{L}$ de ácido tricloroacético com injeção imediata.

O peso da amostra e o volume de ácido tricloroacético foram variáveis importantes na extração, mostrando que a quantidade de amostra deve estar ao redor de 0,9 a $1,1 \mathrm{~g}$ e que o volume de ácido tricloroacético deve ficar na faixa de 300 a $450 \mu \mathrm{L}$ para extração máxima do AF na determinação de ácido fólico em leites enriquecidos.

\section{5 - REFERÊNCIAS BIBLIOGRÁFICAS}

[1] BOX, G. E. P.; HUNTER, W.G; HUNTER, J. S. Statistic for experimenters. An introduction to design, data analysis and model building. Nova York; Wiley, 1978.

[2] BRODY, T. Folic Acid In: MACHLIN L.J. Handbook of vitamins. 2ed. rev. and Expanded. NewYork: Marcel Dekker, 1991.

[3] CATHARINO R.R.; GODOY H. T. Desenvolvimento, validação e aplicação de metodologia para análise de ácido fólico em alimentos enriquecidos. 2000. Dissertação de Mestrado, Faculdade de Engenharia de Alimentos, Universidade de Campinas (UNICAMP).

[4] CRANE, N.T. ; WILSON, D.B. ; COOK, D.A. ; LEWIS, C.J. ; YETLEY, E.A. RADER, J.I. Evaluating food fortification options: general principles revisited with folic acid. American Journal of Public Health, 85 (5): 660-666, 1995.

[5] CZEIZE, A.E.; DUDAS, I. Prevention of the first occurrence of neural tube defects by perioconceptional vitamin supplementetion. New England Journal of Medicine, 327: (26): 1832-1835, 1992.

[6] DALY, S; MILLS, J.R; MOLLOY, A.M; CONLEY, M; LEE, Y.J; KIRKE P.N; WEIR, D.G; SCOTT, J.M. Minimum effective dose of folic acid for food fortification to prevent neuraltube defects. The Lancet, 350:(9092): 1666- 1669, 1997.

[7] DAY, B.P.; GREGORY III, J. F.; Determination of folacin derivatives in selected foods by high-performace liquid chromatography. Journal of Agricultural Food and Chemistry, 29: 374$377,1981$.

[8] DONG, M.W; LEPORE, J; TARUMOTO T. Factors affecting the ion-pair chromatography of water-soluble vitamins. Journal of Chromatography, 442:81-95, 1988.

[9] GREGORYIII, J.F.; SARTAIN, D.B.; DAY, B.P.; Fluorometric determination of folacin in biological materials using high performace liquid chromatography. Journal of Nutrition, 114: 341-353, 1984. 
[10] KATZUNG B.G. Farmacologia básica e clínica. 5ed. São Paulo: Guanabara Koogans, 1994.

[11] KONINGS, E. J. M. A validated liquid chromatographic method for determining folates in vegetables, milk powder, liver, and flour. Jounal of Association of Official Agricultural Chemists International, 82 (1): 119-127, 1999.

[12] LUCOCK, M.D. ; GREEN, M. ; PRIESTNALL, M. ; DASKALAKIS, I. ; LEVENE \&HARTLEY M.I. Optimisation of chromatographic conditions for the determination of folates in foods and biological tisues for nutritional and clinical work. Food Chemistry,53: 329-338, 1995.

[13] MALINOW, M.R. ; DUELL, P.B. ; HESS, D.L. ; ANDERSON, P.H.; KRUGER, W.D. PHILLIPSON, B.E. ; GLUCKMAN, R.A. ; BLOCK, P.C. ; UPSON, B.M. Reduction of plasma homocyst(e)ine levels by breakfast cereal fortified with Folic acid in patients with coronary heart disease. New England Journal of Medicine, 338 (15): 1009-1015, 1998.

[14] MOSHFEGH A.J. ; COOK A.J. ; HO J.W. ; FRIDAY J.E. Folate intakes. Food Surveys Research Group. BHNRC, ARS, USDA, Riverdale, MD, USA, 1998.
[15] OAKLEY, G.P.Jr. ; ERICKSON, J.D. ; ADAMS, M.J. Urgent need to increase folic acid consumption. Jounal of the American Medical Association, 274 (21): 1717-1718, 1995.

[16] RANG, H. P.; RITTER, J. M.; DALE, M. M Farmacologia. 3.ed Rio de Janeiro, Guanabara Koogan, 1997.

[17] SCHULZ, A.; WEIDEMANN, K.; BITSCH, I. Stabilisation of $5-\mathrm{CH}_{3} \mathrm{H}_{4}-\mathrm{Pglu}$ and subsequent analysis by reverse phase high performace liquid chromatography. Journal of Chromatography, 328: 417-421, 1985.

[18] ULENE A.; ULENE V. Vitaminas . 1ed. Blumenau: EKO, 1995.

[19] VAHTERISTO L. T, OLLILAINEN V, KOIVISTOINEN, P. E; VARO P. Improvements in the analysis of reduced folate monoglutamates and folic acid in food by high-performace liquid chromatography. Journal of Agriculture and Food Chemistry, $44:$ :77-482, 1996.

[20] VAHTERISTO L. T, OLLILAINEN V, VARO P. Liquid chromatographic determination of folate monoglutamates in fish, meat, egg, and dairy products consumed in Finland. Journal of Association of Official Analytical Chemists International, 80 (2): 373-378, 1997. 